\title{
Ion Trap Collisional Activation of the Deprotonated Deoxymononucleoside and Deoxydinucleoside Monophosphates
}

\author{
Sohrab Habibi-Goudarzi and Scott A. McLuckey \\ Chemical and Analytical Sciences Division, Oak Ridge National Laboratory, Oak Ridge, Tennessee, USA
}

\begin{abstract}
Deoxymononucleoside and deoxydinucleoside monophosphate anions formed by electrospray have been subjected to ion trap collisional activation. The threshold for decomposition via loss of base is significantly lower for the deoxymononucleoside $3^{\prime}$-monophosphates than for the corresponding $5^{\prime}$-monophosphates, which indicates that the presence of a charged $3^{\prime}$ phosphate group facilitates base loss. The behavior of the bases among each class of isomers shows slight variation in threshold and tandem mass spectrometry efficiency with the notable exception of $2^{\prime}$-deoxyguanosine $5^{\prime}$-monophosphate. This ion is exceptionally stable toward decomposition via base loss, which reflects a strong hydrogen bonding interaction between the base and the phosphate group. All dinucleotides fragment via similar mechanisms, but the propensity for neutral base loss relative to loss of a charged base is highly dependent on the identities of both the $5^{\prime}$ and $3^{\prime}$ bases. The behavior of the dinucleotides under collisional activation conditions supports the proposal that base loss proceeds via a proton-bound dimer intermediate in which loss of the charged base directly competes with loss of the neutral base. Application of the kinetic method allows for quantitative predictions of the differences of the gas-phase acidities of the dimer components. (J Am Soc Mass Spectrom 1995, 6, 102-113)
\end{abstract}

S everal comprehensive reports have appeared that describe the tandem mass spectra derived from anions of the deoxyribomononucleotides and the deoxyribodinucleotides obtained under a wide variety of conditions. These include a study by Cerny et al. [1], wherein decompositions that involve metastable ions and those activated by kiloelectronvolt energy collisions were described, a study of the dinucleotides (both protonated and deprotonated) by Phillips and McCloskey [2] that described results from low energy collisions (21-72 eV laboratory frame) in a hybrid tandem mass spectrometer under multiple collision conditions, and a study of the dinucleotides by Rodgers et al. [3] that described results obtained by using Fourier transform tandem mass spectrometry. The study described here was motivated by the possibility that further light could be shed upon the mechanisms that underlie the decompositions of multiply charged oligonucleotide anions under quadrupole ion trap collisional activation conditions. We have already observed that small multiply charged oligonucleotides fragment via a limited number of channels [4-6] and that the relative contributions of competitive channels can be highly sensitive to the total charge of the ion. A systematic study of the decompositions of the singly

Address reprint requests to Dr. Scott A. Mcluckey, Oak Ridge National Laboratory, P.O. Box 2008, Oak Ridge, Tennessee 37831-6365. charged mono- and dinucleotides under ion trap collisional activation conditions was therefore undertaken to eliminate the influence of internal coulombic repulsion on decomposition so as to highlight its influence in the interpretation of data acquired from multiply charged ions.

Unlike the data reported here, which were acquired on ions derived from electrospray, the published data on the tandem mass spectrometry of mono- and dinucleotide anions have been obtained by using fast-atom bombardment to form the ions [1-3]. The different ionization methods could yield parent ion populations that differ in structural composition and internal energy distribution. Either factor could influence the appearance of the tandem mass spectra. Furthermore, the ion activation conditions in the ion trap are rather unique in that they involve multiple low energy collisions with helium at relatively high frequency $\left(>10^{4}\right.$ $\mathrm{s}^{-1}$ ). This results in a comparatively slow heating process relative to most collisional activation methods [7-10]. Sustained off-resonance irradiation in a Fourier transform mass spectrometer [11] appears to be the collisional activation technique used on the dinucleotides [3] that most resembles the ion trap collisional activation approach employed here.

It was noted in previous studies with multiply charged oligonucleotides that the loss of a nucleobase tends to be the first step in decomposition, followed by 
cleavage at the $3^{\prime} \mathrm{C}-\mathrm{O}$ bond of the sugar from which the base was lost [4-6]. This overall tendency was observed to be independent of charge, but the identity of the bases most likely to be lost and whether or not they were lost as charged or neutral species was observed to be highly dependent on the charge of the parent ion. Of particular interest in this study, therefore, are the tendencies for charged versus neutral base loss, the relative ease with which the various bases are lost, either charged or not, and the importance of the site of phosphorylation, either $5^{\prime}$ or $3^{\prime}$.

\section{Experimental}

All of the mononucleotides and 13 of the dinucleotides used in this study were obtained from Sigma Chemical Co. (St. Louis, MO) as either the free acid or the sodium salt. The dinucleotides $5^{\prime}-\mathrm{d}(G A)-3^{\prime}, 5^{\prime}-\mathrm{d}(\mathrm{GC})-$ $3^{\prime}$, and $5^{\prime}-d(G T)-3^{\prime}$ were synthesized in-house by using a PCR Mate DNA synthesizer (Applied Biosystems, Fountain City, CA). In each case, $10-\mathrm{pmol} / \mu \mathrm{L}$ solutions of $>90 \%$ methanol (remainder water) were prepared and infused at a rate of $1 \mu \mathrm{L} / \mathrm{min}$ through a $120-\mu \mathrm{m}$-i.d. stainless steel needle.

All experiments were carried out with a homemade electrospray source coupled with a Finnigan-MAT (San Jose, CA) ion trap mass spectrometer [12]. Singlefrequency collisional activation via dipolar excitation at $q=$ values of $0.2-0.25$ and irradiation times of $20-50$ ms were used for all parent ions. Helium was present in the vacuum system for all experiments at a pressure of 1 mtorr and air, entering primarily from the interface, was present at $2 \times 10^{-5}$ torr. All tandem mass spectrometry experiments consisted of an ion accumulation period ranging from 50 to $200 \mathrm{~ms}$, an ion isolation step, the collisional activation period, and the mass scan. Roughly $10 \mathrm{~ms}$ were allowed to elapse between the end of the ion isolation period and the beginning of the collisional activation period. The product ion abundances in the tandem mass spectra were insensitive to this delay time, at least beyond a few milliseconds.

\section{Results and Discussion}

\section{Deoxyribomononucleotides}

The tandem mass spectra of the 2'-deoxymononucleoside $3^{\prime}$-monophosphates and the $2^{\prime}$-deoxymononucleoside $5^{\prime}$-monophosphates are summarized in Figures 1 and 2, respectively, which give the abundances of the parent and product ions measured from a series of spectra acquired as a function of resonance excitation amplitude. The parent ion signals are plotted in Figures 1 and 2 to indicate the effiriency with which product ions are formed from the respective parent ions. In many cases, the relative contributions of some of the product ion signals are so low relative to the parent ion signal that their dependence on resonance excitation amplitude cannot be seen. Therefore, Figures 3 and 4 , which plot only the abundances of the product ions, are also included.

By far, the major decomposition channel for all of the 3'-monophosphates is the loss of the neutral base, denoted herein as $\mathrm{BH}$. Minor contributions to the spectra are made by formation of $\mathrm{PO}_{3}^{-}$. The charged base $\left(\mathrm{B}^{-}\right)$was observed for adenine and guanine as well as a few other product ions but in no case did they exceed a few percent of the signal arising from loss of the neutral base. The 5'-monophosphates also show loss of $\mathrm{BH}$ as an important process but the formation of $\mathrm{PO}_{3}^{-}$tends to be much more competitive. (In the case of 2'-deoxycytidine 5'-monophosphate, for example, $\mathrm{PO}_{3}^{-}$is formed in abundance roughly equal to that of the ion formed by base loss, and in the case of 2 '-deoxyguanosine $5^{\prime}$-monophosphate, $\mathrm{PO}_{3}^{-}$gives rise to the base peak in the product ion spectrum at all resonance excitation amplitudes employed in this study.) Water loss following loss of the neutral base also was observed for all of the 5 -monophosphate species; this loss presumably arises from loss of the $3^{\prime}$-hydroxy group along with a hydrogen from the $4^{\prime}$-carbon. This process is analogous to the decomposition sequence observed for the multiply charged oligonucleotides [4-6]. A significant loss of $43 \mathrm{u}$ from $2^{\prime}$-deoxycytidine $5^{\prime}$-monophosphate also was observed. Cerny et al. [1] observed this loss from both the $5^{\prime}$ - and $3^{\prime}$-phosphorylated 2'-deoxycytidines; the 5'monophosphate showed the greatest extent of decomposition through this route. This loss has been attributed to loss of CONH via a retro Diels-Alder mechanism [13]. Several of the $5^{\prime}$-monophosphorylated ions also yielded small signals that corresponded to the formation of $\mathrm{H}_{2} \mathrm{PO}_{4}^{-}$.

Several noteworthy observations can be made from the data of Figures 1-4 that are likely to be relevant in the interpretation of the decomposition behavior of the dinucleotides and larger n-mers. First, in the case of each nucleotide the $3^{\prime}$-monophosphate shows a lower resonance excitation threshold and a greater decomposition efficiency than the corresponding $5^{\prime}$ monophosphate. (The Cerny study [1] pointed out that the ratio of product ion abundances to that of the parent ion in the tandem mass spectra of metastable ions of the $3^{\prime}$-monophosphates was roughly five times greater than that of the corresponding $5^{\prime}$ monophosphates. Although such a ratio does not necessarily reflect the relative kinetic stabilities of the ions, owing to the fact that the internal energy distributions of the ions might not have been the same, it is suggestive that the $5^{\prime}$-isomers have somewhat greater thresholds for decomposition.) The data of Figures 1 and 2 clearly show that the 5 -isomers require more energy to induce fragmentation. A similar conclusion was drawn by Sindona et al. [14] and was attributed to an enhanced acidity of the 2 -hydrogens because of the presence of a charged phosphate group on the 3'carbon. Whatever the mechanism, the presence of the 


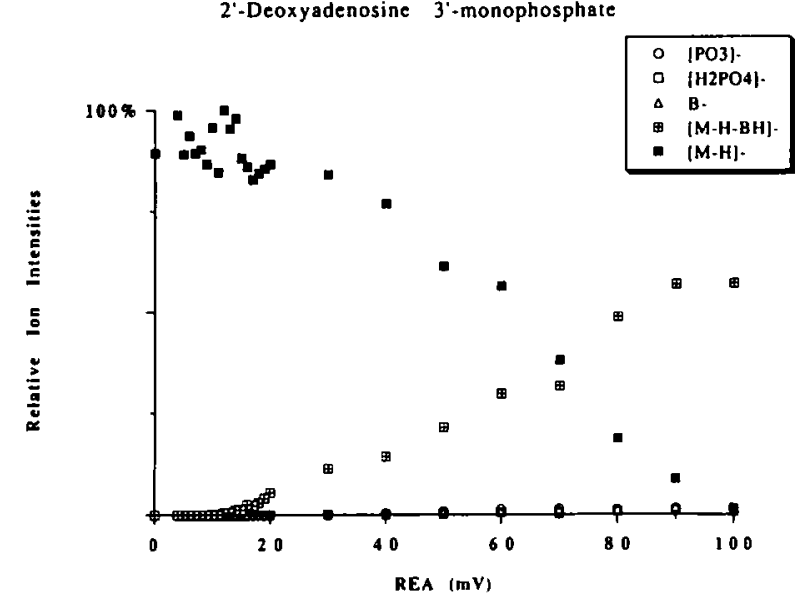

c

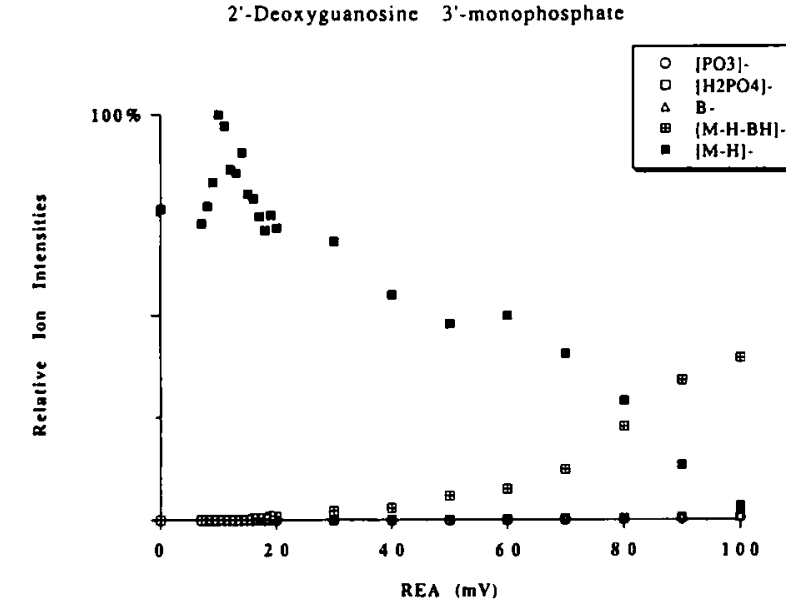

b

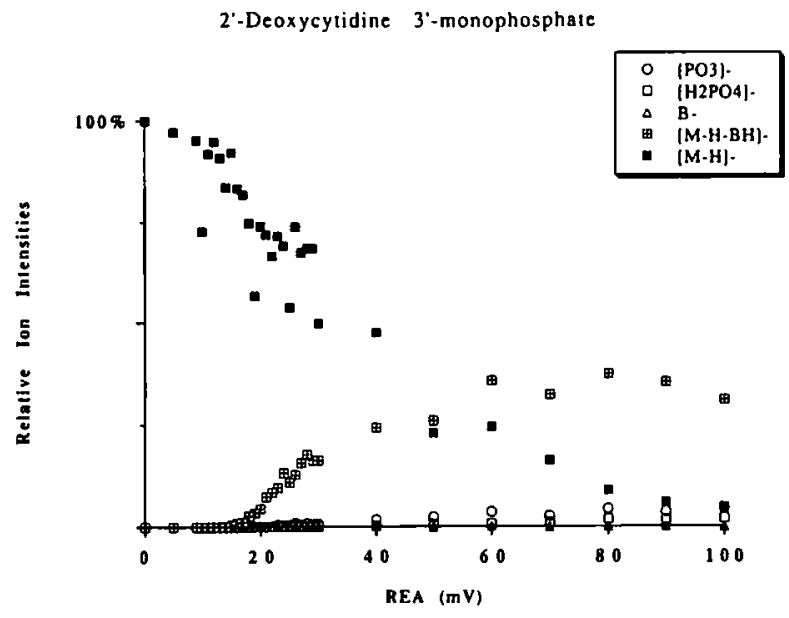

d

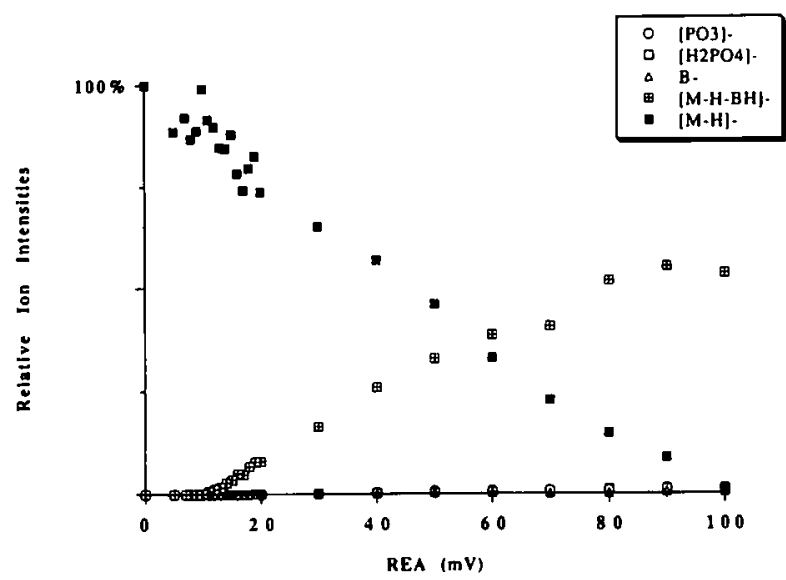

Figure 1. Parent ion and product ion relative intensities acquired as a function of resonance excitation amplitude (REA) for the four deoxymononucleoside $3^{\prime}$-monophosphates.

phosphate group on the $3^{\prime}$-carbon facilitates base loss relative to loss of $\mathrm{PO}_{3}^{-}$.

Among the 3 '-isomers, $\mathrm{Ap}$ and $\mathrm{Tp}$ show roughly similar behavior in that the resonance excitation thresholds for decomposition are comparable and the efficiencies are similar. The $C p$ isomer shows a similar threshold, but the efficiency at higher amplitudes is not as high as those for Ap and Tp. The Gp isomer appears to show a more slowly rising curve for the appearance of the product ions than observed for the other isomers and ultimately shows an efficiency comparable to that of $\mathrm{Cp}$. Although there appear to be no major differences in the likelihood for base loss, the order of facility for neutral base loss among the $3^{\prime}$ isomers appears to be $\mathrm{Ap}, \mathrm{Tp}>\mathrm{Cp}>\mathrm{Gp}$. Among the $5^{\prime}$-isomers, the most dramatic observation is that the pG isomer shows the lowest efficiency for decomposition and a significantly higher threshold for the loss of the neutral base. As previously mentioned, the formation of $\mathrm{PO}_{3}^{-}$is the dominant product for the $\mathrm{pG}$ isomer. The $\mathrm{pC}$ isomer shows slightly higher efficiency and base loss competes roughly equally with formation of $\mathrm{PO}_{3}^{-}$. The $\mathrm{pA}$ and $\mathrm{pT}$ isomers both show higher efficiencies than do the $\mathrm{PG}$ and $\mathrm{pC}$ isomers and both show neutral base loss to be the most facile fragmentation route. Among the 5 -isomers the facility for base loss is therefore $\mathrm{pA}, \mathrm{pT}>\mathrm{pC} \gg \mathrm{pG}$.

Rodgers et al. [3] pointed out in their study of the dinucleotides that the amino group on the 2 position of guanine can hydrogen-bond with a charged phosphodiester linkage when it is present on the $5^{\prime}$-carbon. This interaction can have a significant effect on the loss of a charged base versus a neutral base from the 5 '-base of a dinucleotide (see following text). It seems likely that the possibility for hydrogen bonding between guanine and the phosphate group on the 5 carbon is responsible for the observed enhancement in the stability of $\mathrm{pG}$ as well.

\section{Deoxyribodinucleotides}

Tandem mass spectra were acquired for each of the 16 possible dinucleotides composed of 2'-deoxyadenosine, 2'-deoxyguanosine, 2'-deoxycytidine, and thymidine. Representative results for each of the dinucleotides are shown in Table 1. The structures implied by the shorthand notation used here to represent the various product ions are shown in Scheme I. Under ion 
$2^{\prime}$-Deoxyadenosine $5^{\circ}$-monophosphate

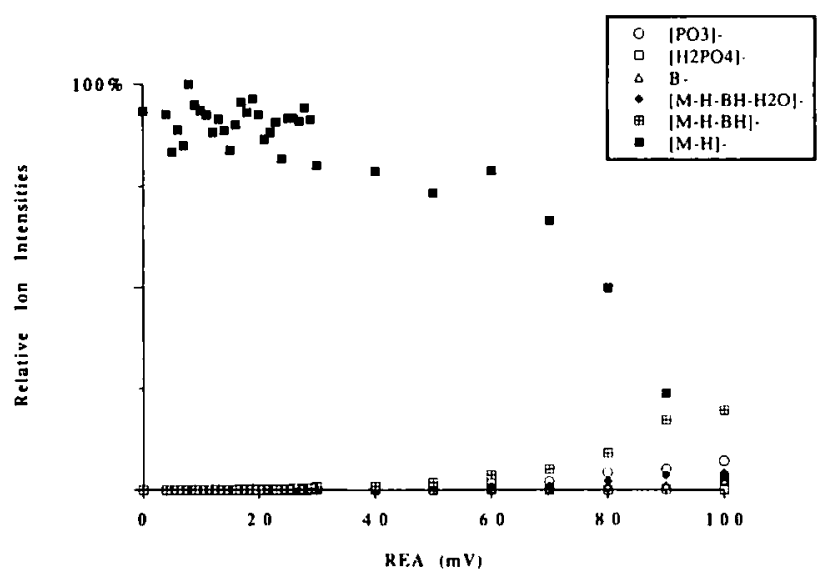

C

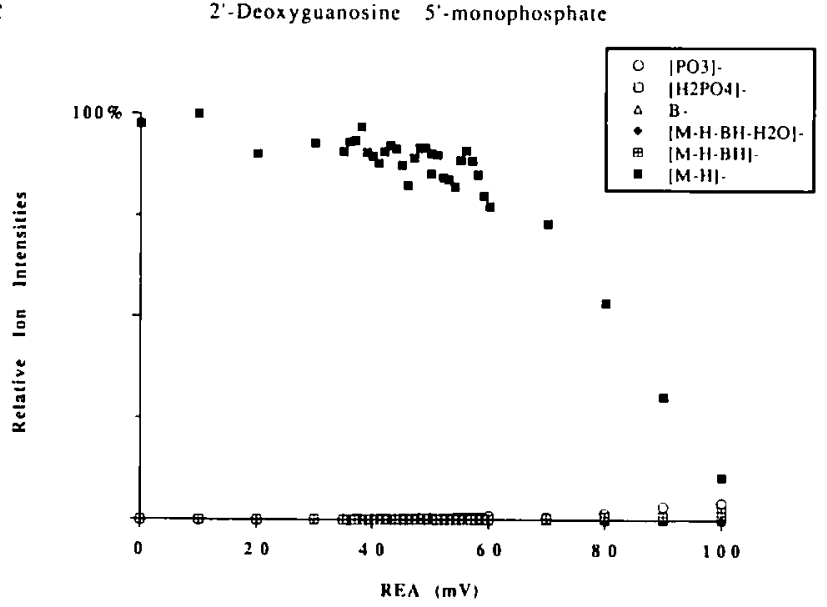

b

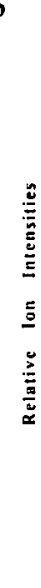

d
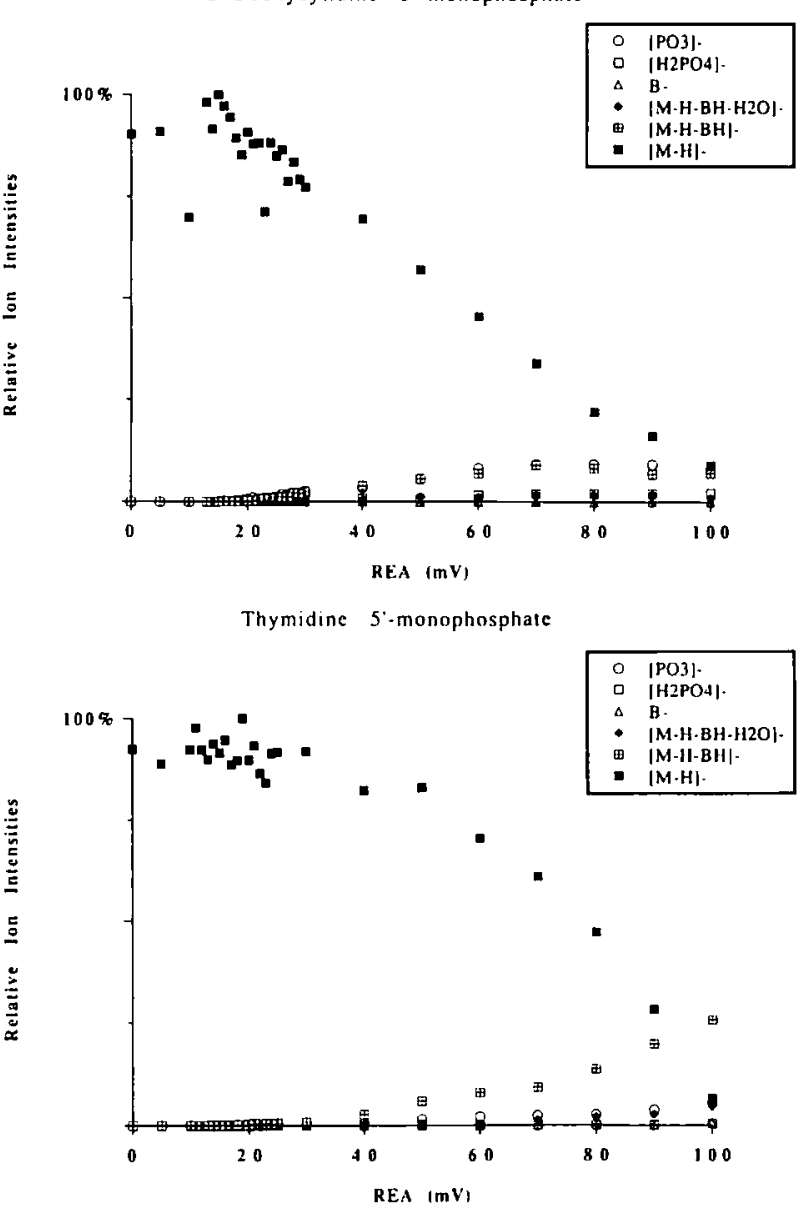

Figure 2. Parent ion and product ion relative intensities acquired as a function of resonance excitation amplitude (REA) for the four deoxymononucleoside $5^{\prime}$-monophosphates.

trap collisional activation conditions, a limited number of dissociation pathways are observed. Previous tandem mass spectrometry studies tended to show a richer variety of product ions, particularly the studies carried out with beam-type tandem mass spectrometers $[1,2]$. The relatively simple tandem mass spectra reported here probably reflect the relatively slow heating nature of ion trap collisional activation. By far the most important dissociation pathway involves loss of the $5^{\prime}$-base either as an ion or as a neutral. Loss of the charged base competes with loss of the neutral base. Subsequent dissociation of the ion formed by loss of the neutral base that results in the loss of the 5 '-sugar is observed in every case. Loss of the $3^{\prime}$-base subsequent to loss of the $5^{\prime}$-sugar is often observed as well. Loss of the 3'-base directly from the parent ion either as an ion or as a neutral occasionally occurs, but is never seen to be a major decomposition pathway. Similar observations were noted in previous tandem mass spectrometry studies of the dinucleotide anions [1-3]. In particular, the ion trap collisional activation results most closely resemble those reported for metastable ions formed by fast-atom bombardment [1] and collisional activation results obtained by using
Fourier transform mass spectrometry [3]. The major competitive and consecutive reactions observed in this study are summarized as follows (see also Scheme I):

$$
\begin{aligned}
& \rightarrow \mathrm{B}_{1}^{-}+\operatorname{sugar}_{1} \mathrm{Sugar}_{2} \mathrm{~B}_{2} \\
& \rightarrow \mathrm{B}_{1} \mathrm{H}+\operatorname{sugar}_{1} \odot^{-} \operatorname{sugar}_{2} \mathrm{~B}_{2}^{-} \\
& \rightarrow \operatorname{sugar}_{1}+\oplus_{\text {sugar }_{2}} \mathrm{~B}_{2}^{-} \\
& \rightarrow \mathrm{B}_{2} \mathrm{H}+\odot^{-} \text {sugar }_{2}^{-} \\
& 5^{\prime}-\left(B_{1} \text { sugar }_{1} \oplus_{\text {sugar }_{2}} B_{2}\right)^{-}-3^{\prime} \\
& \rightarrow \mathrm{B}_{2}^{-}+\mathrm{B}_{1} \text { sugar }_{1} \text { (1) }_{\text {sugar }_{2} \text { (minor) }} \\
& \rightarrow \mathrm{B}_{2} \mathrm{H}+\mathrm{B}_{1} \text { sugar }_{1}{ }^{(} \text {sugar }_{2}^{-} \text {(minor) }
\end{aligned}
$$

Note that further fragmentation of the neutrals in channels (1) and (3) may well take place but the products are not detected. Note also that the sequence shown in channel (2) is a major channel observed for the decompositions of multiply charged oligonucleotides $[4-6,15]$. The other major channel observed for multiply charged oligonucleotides is the analogous 


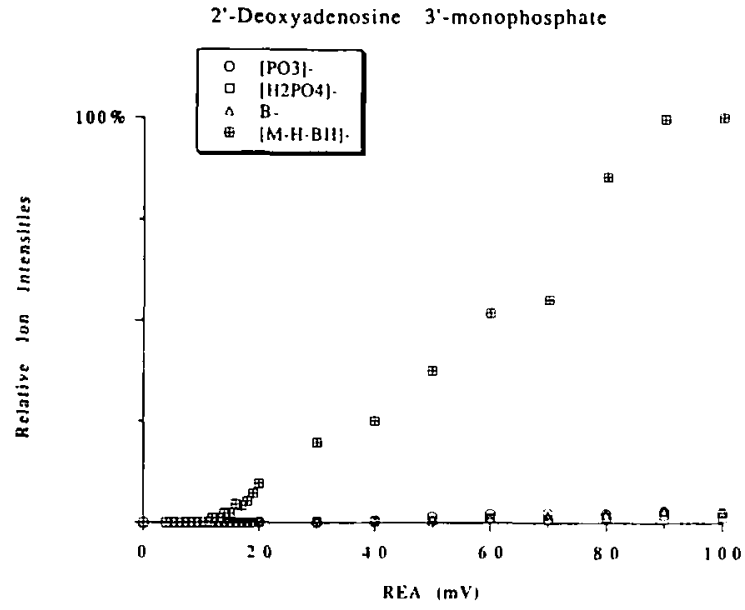

C

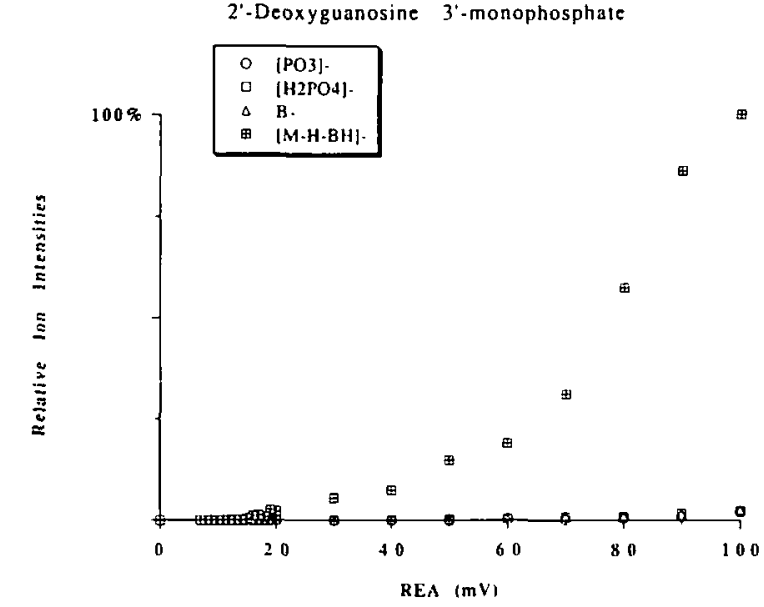

b

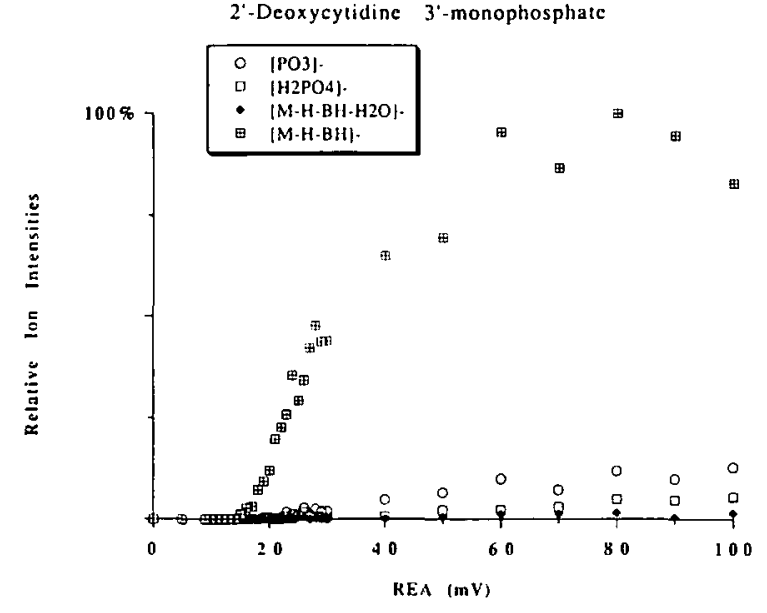

d

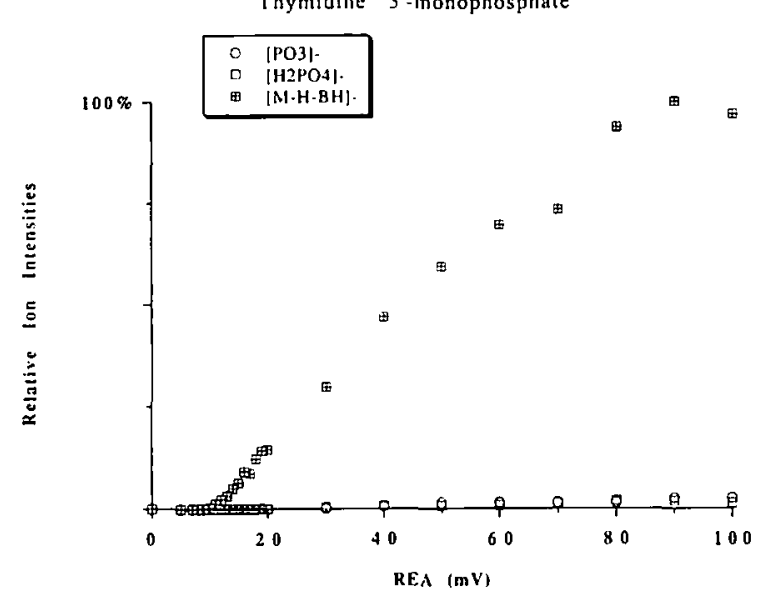

Figure 3. Product ion relative intensities acquired as a function of resonance excitation amplitude for the four deoxymononucleoside $3^{\prime}$-monophosphates.

sequence of dissociations that begin with the loss of a charged base [channel (1)]. The stepwise nature of the dissociation of multiply charged oligonucleotides is directly apparent in the tandem mass spectrometry data because of the fact that both products of a dissociation often carry charge. When this is the case, the complementary nature of product ions is apparent and allows sequential reactions to be identified.

Support for reaction sequence (2) for the singly charged dinucleotides comes from MS" experiments as illustrated in Figure 5. Figure 5a shows the tandem mass spectrum derived from the ion trap collisional activation of the $[\mathrm{M}-\mathrm{H}]^{-}$ion of $5^{\prime}-\mathrm{d}(\mathrm{AT})-3^{\prime}(\mathrm{m} / \mathrm{z}$ 554). Note that the two major peaks correspond to $A^{-}$ $(m / z 134)$ and the loss of $\mathrm{AH}(m / z 419)$. Figure $5 \mathrm{~b}$ shows results of an $\mathrm{MS}^{3}$ experiment with the sequence $m / z 554 \rightarrow m / z 419 \rightarrow$ products. This spectrum shows clearly that collisional activation of the ion formed by the loss of neutral adenine from the parent ion yields a base peak that corresponds to loss of $98 \mathrm{u}$, the mass of the substituted furan formed from the 5 'sugar. The loss of $\mathrm{TH}$ and the loss of water compete with the loss of the remains of the $5^{\prime}$-sugar and the direct loss of $\mathrm{T}^{-}$ also may compete. However, $\mathrm{T}^{-}$can arise also from a consecutive dissociation. In any case, the loss of the $5^{\prime}$-sugar is seen to be the dominant channel. Figure $5 c$ shows the results of an $\mathrm{MS}^{4}$ experiment with the sequence $m / z 554 \rightarrow m / z 419 \rightarrow m / z 321 \rightarrow$ products. In this case, loss of $\mathrm{TH}$ is the dominant channel, which yields an ion at $m / z$ 195, with a lesser degree of loss of $\mathrm{T}^{-}$.

Figure 5 was included here to demonstrate that channel (2) is likely to be the most important route to the formation of many of the product ions in Table 1 under ion trap collisional activation conditions. It already has been demonstrated for multiply charged oligonucleotides in the ion trap $[5,6,15]$ and Rodgers et al. [3] showed the same sequence of reactions for a similar set of $\mathrm{MS}^{n}$ experiments that involved $5^{\prime}$-d(TC)$3^{\prime}$ carried out under Fourier transform mass spectrometry collisional activation conditions. Particular emphasis is placed here on providing support for this sequence of reactions because it is inconsistent with mechanisms for the loss of $\mathrm{BH}$ and the formation of the $m / z 195$ ion originally proposed by Cerny et al. [1] and recently rearticulated by Phillips and McCloskey [2]. The loss of the neutral base has been proposed to result in the formation of a new bond between a 

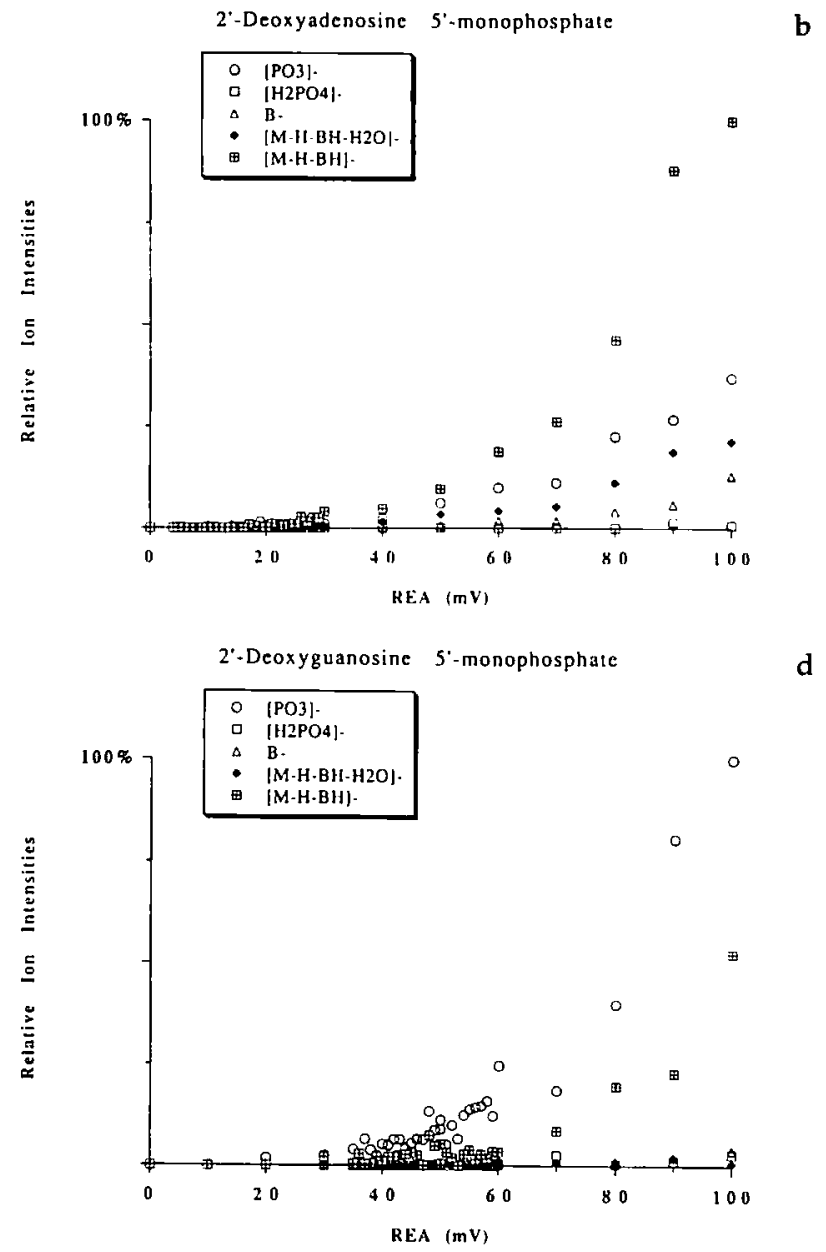

b

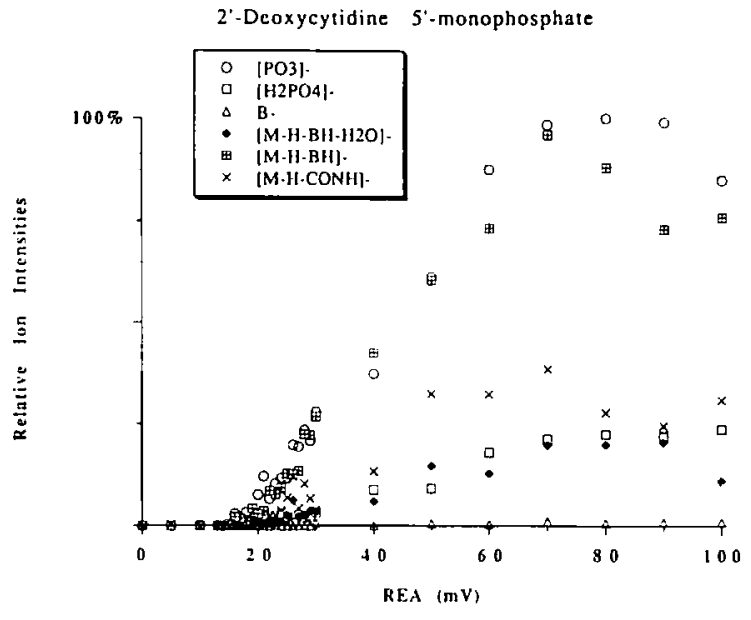

d

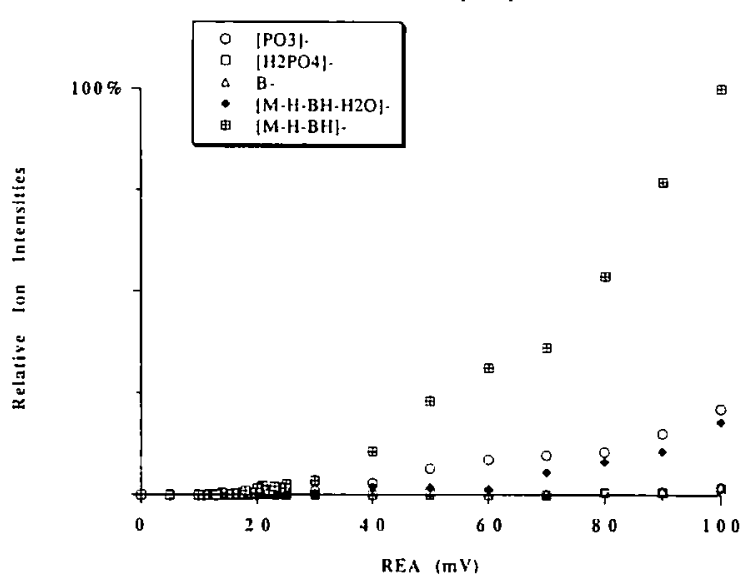

Figure 4. Product ion relative intensities acquired as a function of resonance excitation amplitude for the four deoxymononucleoside 5 '-monophosphates.

Table 1. Relative product ion abundances that result from ion trap collisional activation of the dinucleotides

\begin{tabular}{|c|c|c|c|c|c|c|}
\hline & \multicolumn{6}{|c|}{$\%$ Abundance ${ }^{a}$} \\
\hline & $\mathrm{B}_{1}^{-}$ & $\mathrm{B}_{2}^{-}$ & sugar $_{1} \odot$ sugar $_{2} B_{2}^{-}$ & $\mathrm{B}_{1}$ sugar $_{1} \odot^{\circ}$ sugar $_{2}^{-}$ & $C_{\text {sugar }} B_{2}^{-}$ & (1) sugar $_{2}^{-}$ \\
\hline$\overline{\mathrm{AA}}$ & 100 & & 29 & & & \\
\hline AT & 84 & & 100 & 4 & 24 & 12 \\
\hline$A C$ & 100 & & 81 & 2 & 11 & \\
\hline$A G$ & 7 & 3 & 100 & 3 & 40 & 10 \\
\hline $\mathrm{CA}$ & 3 & 2 & 100 & 5 & 12 & \\
\hline $\mathrm{CC}$ & & & 100 & & 12 & \\
\hline CG & & & 100 & & 33 & 8 \\
\hline CT & & & 100 & 4 & 7 & 15 \\
\hline $\mathrm{GA}^{\mathrm{b}}$ & 100 & & 48 & 6 & 13 & 1 \\
\hline $\mathrm{GC}^{\mathrm{b} . c}$ & 94 & & 100 & 7 & 26 & 3 \\
\hline $\mathrm{GG}$ & 2 & & 100 & & 22 & 4 \\
\hline $\mathrm{GT}^{\mathrm{b}}$ & 46 & & 100 & 15 & 20 & 12 \\
\hline TA & 62 & & 100 & 2 & 11 & \\
\hline TC & 27 & & 100 & & 10 & 2 \\
\hline TG & & & 100 & & 21 & 5 \\
\hline$\pi$ & 6 & & 100 & & 8 & 12 \\
\hline
\end{tabular}

${ }^{a}$ Excludes product ions with abundances less than $1 \%$ of the base peak.

- Includes the abundance of a minor product from further decomposition of $\mathrm{G}^{-}$observed at $\mathrm{m} / \mathrm{z} 133$.

${ }^{c}$ A loss of $43 \mathrm{u}$, presumably due to loss of $\mathrm{CONH}$, also was observed for GC and gave rise to a product

of abundance of $4 \%$. 
GH

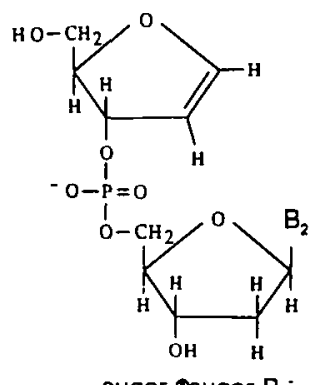

sugar, osugar $_{2} \mathrm{~B}_{2}$.

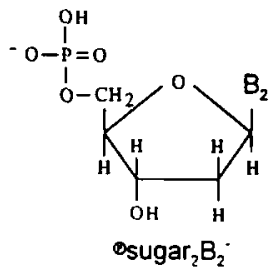

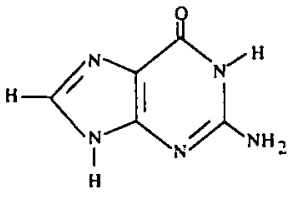

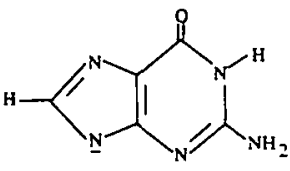

$\mathbf{G}^{*}$

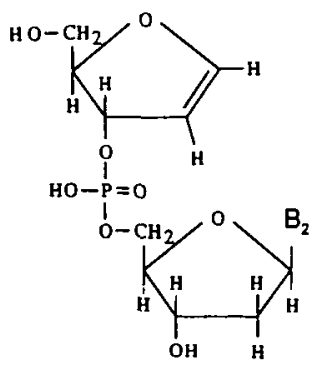

sugar, osugar ${ }_{2} B_{2}$

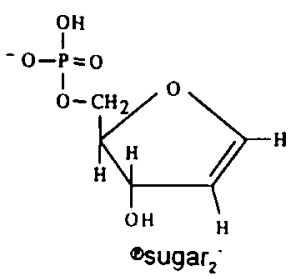

Scheme I

phosphate oxygen and $\mathrm{Cl}^{\prime}$ ' of the deoxyribose, which results in the phosphate group being bound to the deoxyribose at both the $\mathrm{C}^{\prime}$ and $\mathrm{C}^{\prime}$ positions $[1,2]$. Tandem mass spectra of deuterium-labeled parent ions gave results consistent with this mechanism [2]. However, such a product would not be expected to lose readily the proposed furan $[3,5]$ observed to be lost in the $\mathrm{MS}^{3}$ experiment of Figure $5 \mathrm{~b}$ in that two bonds must be cleaved. Cleavage elsewhere along the phosphodiester linkage or loss of the 3'-base would be expected to be much more facile than any mechanism that leads to the loss of $98 \mathrm{u}$ from the proposed structure in which the phosphate group is bound to the sugar at both the $C 1^{\prime}$ and $C 3^{\prime}$ positions $[1,2]$.

The structure of the $m / z 195$ ion was proposed, on the basis of phosphate-C1' closure [2], to contain elements of the 5'-sugar. This structure contrasts with the structure of the $m / z 195$ ion proposed in a study of the high energy collisional activation of singly charged deoxytetranucleotides to be a deoxyribose $5^{\prime}$ phosphate structure with a double bond at C1,2 [16]. The latter structure contains elements of the $3^{\prime}$-sugar. The $\mathrm{MS}^{n}$ results shown here and elsewhere $[3,4-6,15]$ are consistent with the latter structure because it would arise from the major consecutive reaction channel. Both of the mechanisms proposed for the loss of $\mathrm{BH}$ and for the formation of the $\mathrm{m} / \mathrm{z} 195$ ion are fully consistent with the labeling studies [2] once it is recognized that the mechanism outlined here and elsewhere [3, 5] proceeds via consecutive reactions. However, the mechanism described in this paper does not account for the mass shifts observed in the tandem mass spectra of ribose-methylated dinucleotides interrogated by hybrid tandem mass spectrometry under multiple collision conditions [2]. It is important to recognize that the results reported here do not preclude the previously proposed mechanism [1, 2]. Several decomposition channels (mechanisms) may compete, dependent on conditions. However, under the relatively mild collisional activation conditions used in this work, there is no evidence that cleavage of the $5^{\prime} \mathrm{C}-\mathrm{O}$ bond of the $3^{\prime}$-sugar takes place to a significant extent. Rather, the $\mathrm{MS}^{3}$ experiments show cleavage of the $3^{\prime}$ $\mathrm{C}-\mathrm{O}$ bond of the $5^{\prime}$ sugar to be important after loss of the $5^{\prime}$-base.

The identities and locations of the bases in the dinucleotides play important roles in the appearance of the respective tandem mass spectra under all collisional activation conditions thus far reported [1-3] including ion trap collisional activation. In all cases, the $5^{\prime}$-base is lost preferentially, which is consistent with the observation that base loss from the deoxymononucleoside $3^{\prime}$-monophosphates is more facile than base loss from the deoxymononucleoside $5^{\prime}$ monophosphates (see foregoing text). However, the competition between neutral versus charged base loss is highly dependent upon dinucleotide sequence. The data of Table 1 show consistent trends in the likelihood for loss of $B_{1}^{-}$versus loss of $B_{1} H$. Qualitatively, the likelihood for the observance of $B_{1}^{-}$follows the order $A>G>T>C$ for a given $3^{\prime}$-base $\left(B_{2}\right)$. The likelihood for $B_{1}^{-}$loss is greatly affected by the identity of $B_{2}$. The likelihood for the observance of $B_{1}^{-}$proceeds as $B_{2}=A>C>T>G$. The effect of $G$ as the $3^{\prime}$-base is particularly dramatic in its tendency to inhibit formation of $\mathrm{B}_{1}$. It has been pointed out by Rodgers et al. [3] that guanine can readily hydrogen-bond with a charged phosphate group, thereby stabilizing the negative charge on the phosphodiester linkage and reducing the likelihood for proton transfer from the base. The results for the dinucleotides and those for the mononucleotides both support the conclusion that $G$ interacts strongly with a $5^{\prime}$-phosphate group.

Rodgers et al. [3] interpreted the effect of base sequence and identity on the relative propensities for neutral versus charged base loss in terms of competitive decomposition from a common intermediate. The loss of a charged base from the dinucleotides was proposed to result from a proton transfer from the neutral base to the phosphodiester linkage after the base is lost from the $5^{\prime}$-sugar. This process involves the dissociation of an intermediate that can be regarded as a negatively charged proton-bound dimer that consists of the charged base and the phosphodiester group bound to the sugars, that is,

$$
\mathbf{B}_{1}^{-}-\mathrm{H}^{+}-\left(\operatorname{sugar}_{1} \oplus_{\text {sugar }_{2}} \mathrm{~B}_{2}\right)
$$

Several pieces of evidence support the argument that 

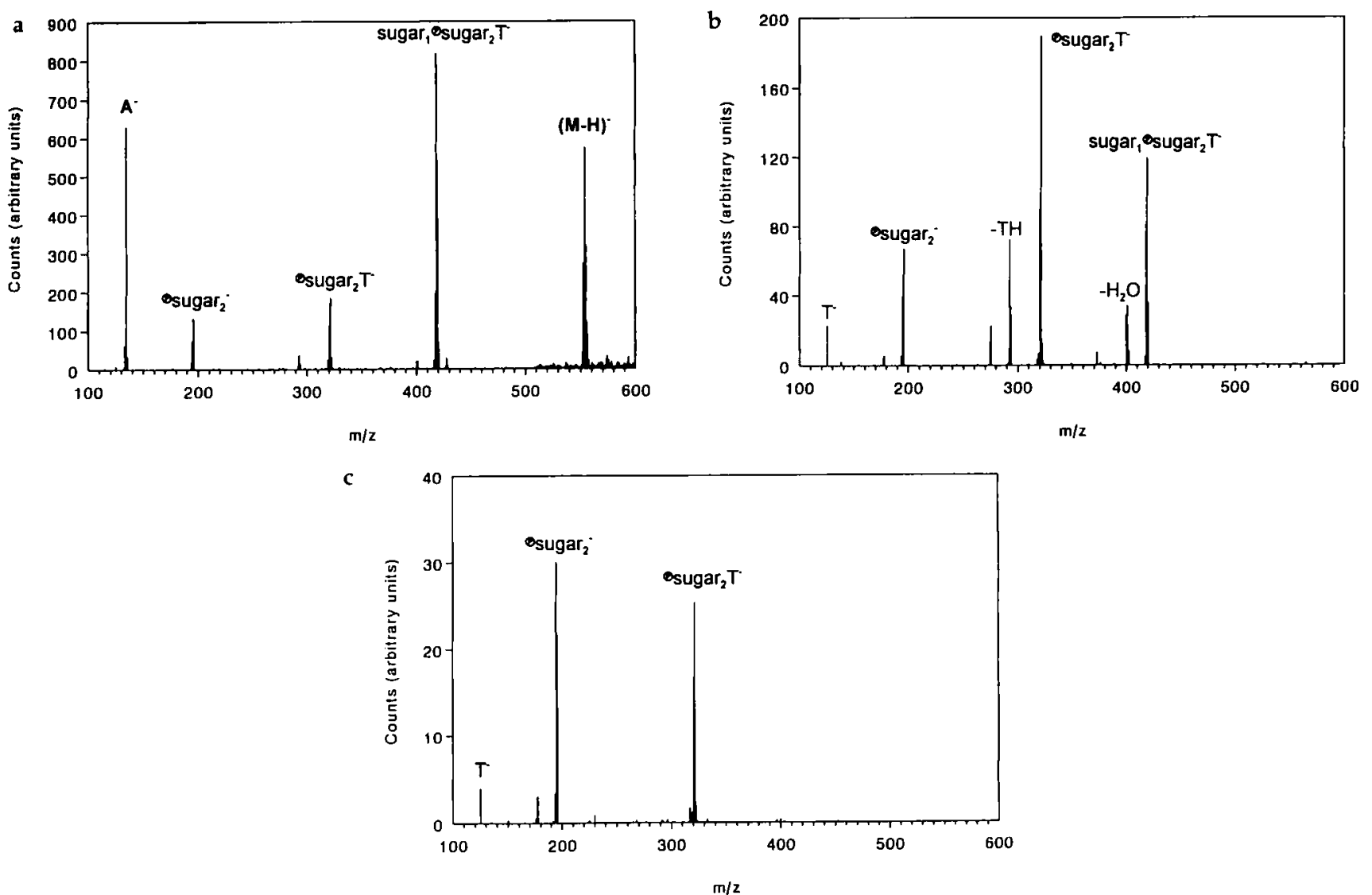

Figure 5. (a) Tandem mass spectrum of the $[M-H]^{-}$parent ion derived from $5^{\prime}-\mathrm{d}(\mathrm{AT})-3^{\prime}$. (b) MS $^{3}$ spectrum derived from the sequence $[M-H]^{-}(m /=554) \rightarrow[M-H-A H]^{-}(m / z+19) \rightarrow$ products. (c) $\mathrm{MS}^{4}$ spectrum derived from the sequence $[\mathrm{M}-\mathrm{H}]^{-}(m /=554) \rightarrow[\mathrm{M}-\mathrm{H}-\mathrm{AH}]^{-}$ $(m / z+19) \rightarrow\left[\mathrm{M}-\mathrm{H}-\mathrm{AH}-\right.$ sugar $^{-}(m / \Sigma 321) \rightarrow$ products.

such a common intermediate is formed in the loss of neutral and charged bases. The $\mathrm{MS}^{\prime \prime}$ results already reported for multiply charged oligonucleotides [5] demonstrated that the loss of a charged base also leads to a decomposition sequence highly analogous to that of sequence (2). (This conclusion can be obtained because the products that arise from loss of $B_{i}$ are often charged and can therefore be interrogated via further stages of mass spectrometry.) The fact that the product ions formed via loss of $\mathrm{B}_{1}^{-}$from multiply charged parent ions fragment very similarly to those formed from loss of $B_{1} H$ suggests that the products are structurally similar and suggests that the two channels proceed over a similar reaction coordinate. A second piece of evidence comes from the internal energy dependence of the ratio of the abundance of $\mathrm{B}_{1}^{-}$to that of the total number of ions that arise from the $B_{1} H$ loss channel. Although the extent to which consecutive reactions occur along reaction channel (2) increases somewhat with resonance excitation amplitude, the ratio of $\mathrm{B}_{1}^{-}$abundance to that of the combined abundances of ions formed from channel (2) is relatively insensitive to resonance excitation amplitude. Very similar results were shown in the Rodgers study [3], which varied collision energy in the Fourier transform mass spectrometry (FTMS) experiment. In both the quadrupole ion trap data (not shown) and the FTMS data, the fraction of product ions comprised of $\mathrm{B}_{1}^{-}$, (the remainder comprised almost exclusively of ions that arise from the $\mathrm{B}_{1} \mathrm{H}$ loss channel) increased slightly with collision energy and then remained constant over a wide range of conditions. Product ion ratios that arise from proton-bound dimers can be highly sensitive to internal energy, particularly near threshold for decomposition [17, 18]. However, it has been shown that at higher internal energies the ratio becomes relatively insensitive to internal energy [19]. The relative independence on ion activation conditions suggests that the internal energy-dependent rates for the competitive reactions are nearly parallel over a wide range of conditions. Such a situation would be highly unlikely for two competitive processes that proceed over substantially different reaction coordinates.

The ion trap data appear to be consistent with the mechanism that involves formation of a proton-bound dimer intermediate in the loss of the base. If this is the case, the tandem mass spectra should then be amenable to treatment by the so-called kinetic method [20-22] to derive semiquantitative estimates of the differences in the free energies of proton transfer between the 
nucleobases and the other ligand of the dimer. In the most approximate formulation for the case of a negatively charged proton bound dimer, the relationship between the ratio of the product ion abundances and the difference in $\Delta G_{\text {acid }}$, the negative of the free energy of protonation of the negatively charged ligand, for each of the ligands is

$$
\ln \left[\mathrm{B}^{-}\right] /[\mathrm{BH} \text { loss channel }] \approx c\left(\Delta \Delta \mathrm{G}_{\mathrm{acid}}\right)
$$

When the entropy changes associated with each channel are equal, the difference in the enthalpies of protonation, that is, the difference in the gas-phase acidities of the ligands, determines the branching ratio, that is,

$$
\ln \left[\mathrm{B}^{-}\right] /[\mathrm{BH} \text { loss channel }] \approx c\left(\Delta \Delta H_{\text {acid }}\right)
$$

Therefore, in such a case the ratio of the relative product ion abundances associated with each competitive decomposition channel can be used to provide an estimate of the difference in gas-phase acidities of the ligands provided the constant $c$ can either be measured or estimated. This constant is usually shown as $1 / R T_{\text {eff }}$, where $T_{\text {eff }}$, the "effective" temperature, is a measure of the internal energy of the fragmenting ions. Ordinarily, the logarithm of the abundance ratio is plotted against either $\Delta G_{\text {acid }}$ or $\Delta H_{\text {acid }}$ by using at least one ligand with a known $\Delta G_{\text {acid }}$ to establish the scale. The slope of the plot is $1 / R T_{\text {eff }} . T_{\text {eff }}$ values for a wide variety of proton-bound dimers have been measured and range from $350-700 \mathrm{~K}$, dependent on collisional activation conditions and the dimer ion. Chemically similar dimers are characterized by a single $T_{\text {eff }}$ under constant experimental conditions [18, 21-23].

The tandem mass spectra of the dinucleotides provide several independent measures of $\Delta \Delta G_{\text {acid }}$ for both the nucleobases and for the species formed by loss of $B_{1}^{-}$(i.e., sugar ${ }_{1}\left(\mathcal{S}_{\text {sugar }_{2}} B_{2}\right.$ ). For example, each set of spectra in which $B_{2}$ is the same provides a ranking of the $\Delta G_{\text {acid }}$ values for the nucleobases. Likewise, each set of spectra with $B_{1}$ held constant provides a ranking of the $\Delta G_{\text {acid }}$ values for the counter ligands. The $\Delta \Delta H_{\text {acid }}$ values obtained for each set of data in which $B_{1}$ is held constant and in which $B_{2}$ is held constant are shown in Tables 2 and 3 . The assumption is made here that the entropies associated with the two competitive dissociations cancel so that the abundance ratios lead directly to gas-phase acidity differences. These values were obtained by using the abundances of $B_{1}^{-}$and the sum of the product ions deemed to arise from the $\mathrm{B}_{2} \mathrm{H}$ loss channel [see channel (2)] shown in Table 1 and assuming $T_{\text {eff }}=400 \mathrm{~K}$. A $T_{\text {eff }}$ of $400 \mathrm{~K}$ was chosen based on previous measures of $T_{\mathrm{eff}}$ for other dimeric species in the ion trap [22] and is expected to be accurate to within $\pm 50 \mathrm{~K}$. The $\Delta \Delta H_{\text {acid }}$ values in Table 2 are indicated as positive or negative relative to the common ligand in the set. Data acquired from the different data sets can be compared directly by using
Table 2. $\Delta \Delta H_{\text {acid }}$ values predicted from the tandem mass

\begin{tabular}{|c|c|c|c|c|}
\hline $\mathrm{B}_{1} \mathrm{~B}_{2}$ & $\begin{array}{c}\ln \left[\mathrm{B}^{-}\right] / \\
{[\mathrm{BH} \text { loss channel] }}\end{array}$ & $\Delta \Delta H_{\text {acid }}{ }^{b}$ & $\mathrm{~B}_{1} \mathrm{~B}_{2}$ & $\Delta \Delta H_{\text {acid }}{ }^{\mathrm{b}}$ \\
\hline$A A$ & 1.24 & 1.0 & AA & 1.0 \\
\hline$A C$ & 0.08 & 0.1 & $C A$ & -2.9 \\
\hline$A G$ & -3.1 & -2.4 & GA & 0.4 \\
\hline AT & -0.48 & -0.4 & TA & -0.5 \\
\hline $\mathrm{CA}$ & -3.6 & -2.9 & $A C$ & 0.1 \\
\hline $\mathrm{CC}^{\mathrm{c}}$ & & & $\mathrm{CC}^{\mathrm{c}}$ & \\
\hline $\mathrm{CG}^{\mathrm{c}}$ & & & $\mathrm{GC}$ & -0.3 \\
\hline $\mathrm{CT}^{\mathrm{c}}$ & & & $\mathrm{TC}$ & -1.1 \\
\hline GA & 0.48 & 0.4 & $A G$ & -2.4 \\
\hline $\mathrm{GC}$ & -0.32 & -0.3 & $\mathrm{CG}^{\mathrm{c}}$ & \\
\hline $\mathrm{GG}$ & -4.1 & -3.3 & $\mathrm{GG}$ & -3.3 \\
\hline GT & -1.1 & -0.8 & $T G^{c}$ & \\
\hline TA & -0.58 & -0.5 & $A T$ & -0.4 \\
\hline TC & -1.42 & -1.1 & $\mathrm{CT}^{c}$ & \\
\hline $\mathrm{TG}^{\mathrm{c}}$ & & & GT & -0.8 \\
\hline$\pi T$ & -2.99 & -2.4 & $\mathrm{TT}$ & -2.4 \\
\hline
\end{tabular}
spectra of the dinucleotides

${ }^{a} \mathrm{BH}$ loss channel includes signals due to sugar ${ }_{1}\left(\mathcal{C}_{\text {sugar }_{2}} \mathrm{~B}_{2}\right.$.

P $_{\text {sugar }}{ }_{2} B_{2}^{-}$, and Sugar $_{2}^{-}$listed in Table 1.

${ }^{\mathrm{b}} \Delta H_{\mathrm{acid}}$ values are listed in units of kilocalories per mole and are determined with an assumed $T_{\text {efl }}$ of $400 \mathrm{~K}$ (see text).

${ }^{c}$ Signal for $\mathrm{B}^{-}$was too small for a reliable ratio measurement.

Table 3. $\Delta \perp H_{\text {acid }}$ values $(\mathrm{kcal} / \mathrm{mol})$ relative to

\begin{tabular}{|c|c|}
\hline Species & $\Delta \Delta H_{\text {acid }}$ \\
\hline sugar $_{1}(1)$ & $<-2.4$ \\
\hline sugar $_{1}\left(\right.$ sugar $_{2} G$ & -2.4 \\
\hline sugar $_{1}{ }^{\mathbb{C}}$ sugar $_{2} \mathrm{~T}$ & -0.4 \\
\hline A & $\equiv 0$ \\
\hline sugar $_{1}\left(\right.$ sugar $_{2} \mathrm{C}$ & 0.1 \\
\hline $\mathrm{G}$ & 0.6 \\
\hline sugar $_{1}\left({ }^{(1)}\right.$ sugar $_{2} A$ & 1.0 \\
\hline$T$ & 1.5 \\
\hline C & 3.9 \\
\hline
\end{tabular}
that of adenine"

${ }^{a}$ Values for the nucleobases are taken from measurements in which sugar, $\mathbb{C}_{\text {sugar }} A^{-}$was the counter ligand. Values for the sugar, (1) sugar ${ }_{2} B_{2}$ species are taken from measurements in which $A^{-}$was the counter ligand.

the relative $\Delta \Delta H_{\text {acid }}$ values determined by assigning $\Delta H_{\text {acid }}$ (adenine) $=0$ (Table 3).

Although each tandem mass spectrum provides an independent comparison of the relative acidities of the ligands, some provide much more reliable quantitative estimates than others. Large or small abundance ratios lead to increased possibility for error because of the increased relative error in the abundance measurement of a small peak. For this reason, the data acquired with $\mathrm{B}_{1}=\mathrm{C}$ are less valuable for semiquantitative measurements because the low apparent acidity of cytidine results in very small $\mathrm{C}^{-}$abundances. Likewise, the data acquired for $B_{2}=G$ are of limited value for 
quantitative ranking of the acidities of the nucleobases due to the high apparent acidity of ( $\operatorname{sugar}_{1} \odot^{-}$sugar $_{2} G$ ), which leads to small abundances for $B_{1}^{-}$. Possible sources of error in the ion trap measurements for all of the dinucleotides may arise from instrumental discrimination effects. For example, the other tandem mass spectrometry studies showed formation of $\mathrm{PO}_{3}^{-}$under some conditions. The $q_{z}$ values used in these studies for the parent ions did not allow for trapping ions of $m / z$ 79. This ion is expected to be formed from (1) sugar $_{2} B_{2}^{-}$[see reaction channel (2)] in competition with loss of $\mathrm{B}_{2} \mathrm{H}$ to yield ${ }^{-}$sugar $_{2}^{-}$and as a consecutive decomposition product from ${ }^{\circ}$ sugar $_{2}^{-}$. Any $\mathrm{PO}_{3}^{-}$ formed from either route would lead to an underestimate of the $\mathrm{B}_{2} \mathrm{H}$ loss channel. The storage efficiency also is expected to vary over the mass-to-charge range of the product ions. In particular, the $\mathrm{B}_{1}^{-}$ions are expected to be discriminated against because they are formed at relatively high $q$ : values under the conditions used here. Discrimination against $B_{1}^{-}$results in an underestimate of the $B_{1}^{-}$loss channel (i.e., an error opposing any error due to formation of $\mathrm{PO}_{3}^{-}$). No concerted effort was made here to sort out the magnitudes of these possible error sources. However, it is important to note that in all cases at least $60 \%$ of the parent ions could be accounted for by formation of product ions. The difference $(<40 \%)$ is expected to be comprised of loss of parent ions because of resonance ejection in addition to any product ion losses. Therefore, because the major potential sources of error tend to cancel and because high tandem mass spectrometry efficiencies are observed, the values given here are expected to fall within $\pm 0.2 \mathrm{kcal} / \mathrm{mol}$ for the differences between $A$ and $G$. Somewhat larger errors can be anticipated for the acidity differences with cytosine and with the sugar ${ }_{1}{ }^{-}$sugar $_{2} G_{2}$ ligand because of the relatively small $B_{1}^{-}$ions formed with dinucleotides in which $B_{1}=C$ and $B_{2}=G$.

Several noteworthy observations are suggested by the data of Tables 2 and 3 . One is that the $\Delta H_{\text {acid }}$ (adenine) and $\Delta H_{\text {acid }}$ (guanine) are similar and that of guanine exceeds that of adenine by about 0.6 $\mathrm{kcal} / \mathrm{mol}$. The $\Delta H_{\text {acid }}$ of thymine exceeds that of adenine by about $1.5 \mathrm{kcal} / \mathrm{mol}$ and the $\Delta H_{\text {acid }}$ of cytosine is greater than that of adenine by $3.5-4.5 \mathrm{kcal} / \mathrm{mol}$. Tables 2 and 3 indicate that the $\operatorname{sugar}_{1} \odot_{\text {sugar }_{2}} B_{2}$ species have $\Delta H_{\text {acid }}$ values on the order $\mathrm{B}_{2}=\mathrm{A}>\mathrm{C}$ $>\mathrm{T} \gg$ G. Roughly $0.9 \mathrm{kcal} / \mathrm{mol}$ separates $B_{2}=A$ and $B_{2}=C$ and roughly $1.4 \mathrm{kcal} / \mathrm{mol}$ separates $B_{2}=A$ and $B_{2}=T$. As expected, the $B_{2}=G$ is well over 3 $\mathrm{kcal} / \mathrm{mol}$ more acidic than the $B_{2}=A$ species.

It is also noteworthy that the loss of a charged 5 '-base is sometimes observed to be a major decomposition pathway for the dinucleotides, whereas loss of a charged base is not observed to be a major process for any of the mononucleotides. The proton-bound dimer intermediate for an analogous mechanism for the $3^{\prime}$-mononucleotides, for example, replaces the $3^{\prime}$ - nucleoside with a hydrogen, that is,

$$
\mathrm{B}^{-}-\mathrm{H}^{+}-\text {sugar }()^{-}
$$

The acidity of sugar ${ }^{\odot}$ relative to the sugar ${ }_{1} \odot_{\text {sugar }} \mathrm{B}_{2}$ species is revealed by comparing the $\left[\mathrm{B}^{-}\right] /[\mathrm{BH}$ loss channel] ratio for the $3^{\prime}$-mononucleotides with the relevant dinucleotide results for constant $B_{1}$. For example, the data for $\mathrm{Ap}^{-}$is compared with the data for the $\mathrm{ApB}_{2}^{-}$ions. In each case, the relative lack of $\mathrm{B}_{1}^{-}$ formation from the $3^{\prime}$-mononucleotides suggests that the acidity of sugar $\left({ }^{-1}\right.$ is greater than that of sugar $_{1} \oplus_{\text {sugar }} B_{2}$, where $B_{2}=A, T, C$. The relative lack of $B_{1}^{-}$loss in the dinucleotides in which $B_{2}=G$ makes a ranking of sugar $(5$ relative to sugar $_{1}\left(\oplus_{\text {sugar }_{2}} \mathrm{G}\right.$ difficult. However, greater extents of $A^{-}$and $G^{-}$formation are observed from $5^{\prime}-d(A G)-3^{\prime}$ and $5^{\prime}-\mathrm{d}(\mathrm{GG})-3^{\prime}$, respectively, than from $A p$ and $\mathrm{Gp}$, which suggests that the acidity of sugar ${ }^{\circledR}$ is also greater than that of $\operatorname{sugar}_{1} \odot_{\text {sugar }_{2}} \mathrm{G}$. Unfortunately, the small $\left[\mathrm{B}^{-}\right] /[\mathrm{BH}$ loss channel] ratios noted for the 3'-mononucleotides do not allow for a reliable quantitative $\Delta \Delta H_{\text {acid }}$ comparison. Nevertheless, these results suggest that the presence of a 3 '-nucleoside destabilizes the negative charge on the phosphate group and thereby increases the $\Delta H_{\text {acid }}$ values of the sugar $_{1}{ }^{(}$sugar $_{2} B_{2}$ species relative to that of sugar $\left.{ }^{(}\right)$. The strong hydrogen bonding interaction apparent for $B_{2}=G$ clearly stabilizes the charge such that sugar $_{1}\left({ }^{-}\right.$sugar $_{2} G$ has the closest gas-phase acidity to that of sugar ${ }^{\odot}$ of all of the sugar ${ }_{1}{ }^{-}$sugar $_{2} B_{2}$ species in this study.

Similar arguments can be used to conclude that the acidity of 1 sugar, formed from the 5'-mononucleotides, is greater than that of any of the nucleobases and for most of the sugar ${ }_{1} \odot_{\text {sugar }} B_{2}$ species. This conclusion is again based on the small $\left[\mathrm{B}^{-}\right] /[\mathrm{BH}$ loss channel] ratios observed from the $5^{\prime}$-mononucleotides. Somewhat greater $\mathrm{B}_{1}^{-}$signals are observed from the 5 '-mononucleotides than from the $3^{\prime}$-mononucleotides. However, it is probably not justifiable to conclude that there is a measurable difference between the acidities of ${ }^{-}$sugar and sugar ${ }^{\circ}$. Cleavage of the base is presumed to precede formation of the proton-bound dimer, and the plots of Figures 1-4 demonstrate that more energy is required for this process for the $5^{\prime}$ nucleotides than for the 3 '-nucleotides. Therefore, the internal energies of the species being sampled may differ such that there could be a significant difference in the $T_{\text {eff }}$ values for the $3^{\prime}$-mononucleotides and the dinucleotides, which all involve cleavage of a base with a $3^{\prime}$-phosphate, versus the 5 '-mononucleotides, which involve cleavage of a base with a $5^{\prime}$-phosphate. Therefore, a direct comparison of the $\mathrm{pA}$ data, for example, with the data for the $B_{1} p A$ species is not as justifiable as the comparison of the Ap data with the data for the $\mathrm{ApB}_{2}$ species.

Rodgers et al. [3] concluded an ordering for the 
gas-phase acidities of the nucleobases from their experimental data to be $A<T<G<C$ and reported an order based on semiempirical calculations of $T<G<$ $A<C$. It was suggested that the calculations may not be sufficiently accurate to differentiate between the gaseous acidities of $T, G$, and $A$. The experimental ranking differs from that reported here in that $T$ and $G$ are reversed. However, upon analyzing the data reported in the paper of Rodgers et al. [3] as the data were analyzed here, by comparing $\left[\mathrm{B}^{-}\right] /[\mathrm{BH}$ loss channel] ratios, the dinucleotides in which $B_{2}=C, T$ give the order $A<G<T<C$, in agreement with the ranking reported here. The dinucleotides in which $B_{2}$ $=A$ suggest the order $A<T<G<C$. It appears as though the relative abundance of $\mathrm{T}^{-}$is consistently greater in the FTMS data than in the ion trap data reported here. It is not clear why this should be so, but the possibility for discrimination against the relatively low mass-to-charge $\mathrm{T}^{-}$anion in the ion trap might account for this difference. Such a systematic error would lead to an overestimate of the gas-phase acidity of thymine reported here and would put its value closer to that of guanine.

It is important to emphasize that the interpretations given here are based on the assumption that the ions decompose via a common proton-bound dimer intermediate. These results do not rule out other mechanisms that might give rise to an apparent gas-phase acidity ordering that differs from the true order. For example, a reviewer has pointed out the possibility that loss of $\mathrm{T}^{-}$might be disfavored because of interaction of the oxygen on the 2 position of the thymine ring with the hydrogens on the 2 ' position of the sugar. Such an interaction could change the apparent acidity order.

\section{Conclusions}

The behavior of singly charged deoxymono- and deoxydinucleotides under ion trap collisional activation conditions reveals several phenomena that should be helpful in interpreting larger multiply charged oligonucleotides. The mononucleotide data indicate that the loss of the base is significantly facilitated by the presence of a $3^{\prime}$-phosphate group as opposed to a 5 '-phosphate group. Among each set of mononucleotides the facility for base loss follows the trend Ap, $\mathrm{Tp}>\mathrm{Cp}>\mathrm{Gp}$ for the $3^{\prime}$-monophosphates and $\mathrm{pA}$, $\mathrm{pT}>\mathrm{pC} \gg \mathrm{pG}$ for the $5^{\prime}$-monophosphates. The significantly greater threshold for decomposition of $p G$ is probably attributable to strong hydrogen bonding between the amine group on guanine and the phosphate group [3].

All of the dinucleotides show similar decomposition mechanisms in which loss of a charged base from the $5^{\prime}$ end of the molecule competes with loss of the neutral base. Consecutive reactions that follow base loss involve loss of the elements of the $5^{\prime}$-sugar fol- lowed by loss of the $3^{\prime}$-base or loss of $\mathrm{PO}_{3}^{-}$in analogy with the mechanisms identified for multiply charged oligonucleotides [5]. However, the extent to which the $5^{\prime}$-base is lost as an ion or as a neutral is highly dependent on the identity of the $5^{\prime}$-base as well as the identity of the 3'-base. The decomposition behavior of the dinucleotide anions as a function of resonance ejection amplitude is consistent with loss of a neutral or a charged base proceeding through a common proton-bound intermediate [3]. If this is so, application of the kinetic method to rank gas-phase acidities can yield quantitative predictions of the differences in $\Delta H_{\text {acis }}$ of the nucleobases as well as the sugar $\odot_{\text {sugar }} B_{2}$ species. Qualitatively, the gas-phase acidities of the nucleobases follow the order $A<G<$ $\mathrm{T}<\mathrm{C}$, whereas the acidities of the sugar $_{1} \odot$ sugar $_{2} \mathrm{~B}_{2}$ species follow the order $\mathrm{B}_{2}=\mathrm{A}>\mathrm{C}>\mathrm{T} \gg \mathrm{G}$. Comparison of the mononucleotide data with the dinucleotide data suggests that the $\Delta H_{\text {acid }}$ value of the sugar (1) group is significantly lower than those of the nucleobases as well as those of the sugar ${ }_{1}$ sugar $_{2} B_{2}$ species.

\section{Acknowledgments}

The authors thank Dr. J. L. Beauchamp for a copy of ref 3 prior to publication. This work was supported by the National Institutes of Health under Grant R01 GM45372. Oak Ridge National Laboratory is managed for the U.S. Department of Energy under Contract DE-AC05-84OR21400 by Martin Marietta Energy Systems, Inc. SHG acknow'ledges support through an appointment to the Oak Ridge National Laboratory Postdoctoral Research Associates Program administered jointly by the Oak Ridge Institute of Science and Education and Oak Ridge National Laboratory.

\section{References}

1. Cerny, R. L.; Ciross, M. L.; Grotjahn, L. Amal. Biochem. 1986, $1.56,424$

2. Phillips, D. R.; McCloskey, J. A. Int. I. Mass Spectrom. Ion Procissies 1993, 128, 61.

3. Rodgers, M. T.; Campbell, S.; Marzluff, E. M.; Beauchamp, J. L. Int. I. Mass Spectrom. 1994, 137, 121

4. McLuckey, S. A.; Habibi-Goudarzi, S. I. Am. Sor. Mass SpeiIrom. 1994, 5, 740.

5. McLuckey, S. A.; Habibi-Goudarzi, S. 1. Am. Ch'm. Soc. 1993, $115,12085$.

6. McLuckey, S. A.; Van Berkel, G. J.; Glish, G. L. I. Am. Sor. Mass Spectrom. 1992, 3, 60.

7. March, R. E. Int. I. Mass Spectrom. lon Processes 1992, $118 / 119,71$.

8. Gronowska, J.; Paradisi, C.; Traldi, P.; Vettori, U. Rapid Commun. Mass Spectrom. 1990, 4, 306.

9. Hart, K. J.; McLuckey, S. A. I. Am. Soc. Mass Spectrom. 1994, 5,250

10. Mcluckey, S. A. I. Am. Soc. Mass Spictrom. 1992, 3, 599.

11. Gauthier, J. W.; Trautman, T. R.; Jacobson, D. B. Anal. Chim. Acta 1991, 246, 211.

12. Van Berkel, G. J.; Glish, G. L.; McLuckey, S. A. Anal. Ch'm. $1990,62,1284$. 
13. Neri, N.; Sindona, G.; Uccella, N. Gazz. Chim. Ital. 1983, 113, 197.

14. Sindona, G.; Uccella, N.; Weclawek, K. J. Chem. Res. (Suppl.) 1982, 184.

15. Little, D. P.; Chorush, R. A.; Speir, J. P.; Senko, M. W.; Kelleher, N. L.; McLafferty, F. W. J. Am. Chem. Soc. 1994, 116, 4893.

16. Panico, M.; Sindona, G.; Uccella, N. J. Am. Chem. Soc. 1983, $105,5607$.

17. Wright, L. G.; McLuckey, S. A.; Cooks, R. G.; Wood, K. V. Int. J. Mass Spectrom. Ion Phys. 1982, 42, 115.
18. McLuckey, S. A.; Cooks, R. G.; Fulford, J. E. Int. I. Mass Spectrom. Ion Phys. 1983, 52, 165.

19. McLuckey, S. A. Org. Mass Spectrom. 1984, 19, 545.

20. Cooks, R. G.; Kruger, T. L. J. Am. Chem. Soc. 1977, 99, 1279.

21. McLuckey, S. A.; Cameron, D.; Cooks, R. G. J. Am. Chem. Soc. 1981, 103, 1313.

22. Patrick, J. S.; Kotiaho, T.; McLuckey, S. A.; Cooks, R. G. Mass Spectrom. Rev., accepted for publication.

23. Nourse, B. D.; Cooks, R. G. Int. I. Mass Spectrom. Ion Processes 1991, 106, 249. 